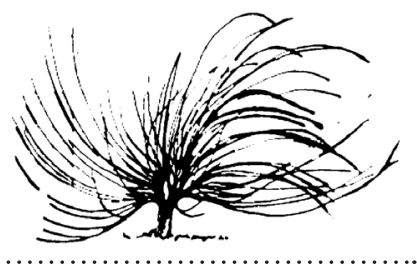

\title{
Pedagogía decolonial: hacia la configuración de biopraxis pedagógicas decolonizantes
}

\author{
Alexander Luis Ortiz Ocaña ${ }^{2}$ \\ Universidad del Magdalena \\ Colombia \\ alexanderortiz2009@gmail.com \\ María Isabel Arias López ${ }^{3}$ \\ Institución Educativa Departamental de Tucurinca \\ Colombia \\ mariaisarias2015@gmail.com \\ Zaira Esther Pedrozo Conedo ${ }^{4}$ \\ Institución Educativa Departamental Thelma Rosa Arévalo-Varela \\ Colombia \\ zairapedrozoc@gmail.com
}

(c) $\left(\begin{array}{l}\mathrm{BY} \\ \mathrm{NC}\end{array}\right.$

Recibido: 28 de abril de 2018. Aprobado: 24 de setiembre de 2018.

http://dx.doi.org/10.15359/rep.13-2.10

1 Artículo de revisión derivado del proyecto de investigación financiado por FONCIENCIAS, Universidad del Magdalena, Santa Marta, Colombia (Proyecto escenarios formativos mediadores de las biopraxis de niños y niñas en contexto de pobreza).

2 Doctor en Ciencias Pedagógicas, Universidad Pedagógica de Holguín, Cuba. Doctor Honoris Causa en Iberoamérica, Consejo Iberoamericano en Honor a la Calidad Educativa (CIHCE), Lima, Perú. Magíster en Gestión Educativa en Iberoamérica, CIHCE, Lima, Perú. Magíster en Pedagogía Profesional, Universidad Pedagógica y Tecnológica de la Habana. Licenciado en Educación. Recibió el premio a la excelencia educativa 2007 y 2008, otorgado por el CIHCE con sede en Lima, Perú. Mejor pedagogo novel de Cuba en el 2002. Ha realizado asesorías pedagógicas, talleres y conferencias en Cuba, México, Brasil, Ecuador, Venezuela, Panamá, Chile, Paraguay y Colombia.

3 Especialista en Pedagogía, Cultura Constitucional y Democracia, Universidad Autónoma de Colombia. Licenciada en Ciencias Sociales de la Universidad del Magdalena, Santa Marta, Colombia. Magíster en Educación SUE Caribe, en dicha universidad. Ha sido docente catedrática en la Universidad del Magdalena, Santa Marta, Colombia. Docente tutora en la Universidad de Pamplona a Distancia, Santa Marta, Colombia. Docente en los niveles de básica primaria, básica secundaria y media en Santa Marta, Colombia.

4 Especialista en Ética y Pedagogía, de la Corporación Universitaria Juan de Castellano. Psicóloga social comunitaria. Magíster en Educación de la Universidad del Magdalena, Santa Marta, Colombia. Experta en el abordaje de habilidades y valores para planear, prevenir, diagnosticar, intervenir, evaluar e investigar, en el ámbito del comportamiento y la educación. Participa y colabora en equipos de trabajo interdisciplinarios en el manejo de los distintos enfoques y modelos de aprendizaje, estrategias de enseñanza, motivación, estrategias para el manejo del salón de clases, proyectos de aula, métodos de evaluación, formación por competencias, entre otros. Asistente a congresos, talleres y cursos afines al área. Conferencista, consultora y asesora tanto psicológica como pedagógica de instituciones educativas y comunidades. 


\title{
Resumen
}

El giro decolonial se ha preocupado más por la cuestión ideopolítica, epistémica y epistemológica que por la problemática disciplinar (pedagógica, curricular y didáctica), y su ocupación en lo relacionado con la evaluación de los aprendizajes es casi nula. No se ha trabajado con amplitud y profundidad la decolonialidad de la educación. Es necesario decolonizar el sistema categorial de la pedagogía, el currículo y la didáctica. Urge hacer una lectura decolonial de la genealogía de las ciencias educativas. Mirar la pedagogía, el currículo y la didáctica en clave decolonial nos permitirá develar el carácter USAeurocéntrico del sistema categorial de las teorías de la educación y la enseñanza. Debemos desoccidentalizar las ciencias de la educación y develar la doctrinalidad de la educación y la formación, el proceso que oculta la pedagogía en su propuesta de la noción de formación, así como la contradicción entre formación y emancipación. La doctrinalidad es la cara oculta de la formación.

Palabras claves: giro decolonial, decolonialidad de la educación, pedagogía, currículo, didáctica, ciencias de la educación.

\begin{abstract}
The decolonial turn is more concerned about issue of ideopolitics, epistemics, and epistemology than problems by discipline (pedagogical, curricular and didactic), and its preocupation with the evaluation of learning outcomes is almost non-existent. We have not worked with the breadth and depth of decoloniality in education. It is necessary to decolonize the categorical system of pedagogy, curriculum, and didactics. It is urgent to make a decolonial reading of the genealogy of Educational Sciences. Looking at pedagogy, curriculum, and didactics from a decolonial viewpoint will allow us to uncover the Eurocentric nature of the categorical system of these theories of education and teaching. We must de-link Educational Sciences from Eurocentricity and reveal the doctrinality of education and training, the process that pedagogy hides in its proposal
\end{abstract}


for the notion of training as well as the contradiction between training and emancipation. Doctrinality is the hidden side of the training.

Keywords: decolonial turn, decoloniality of education, pedagogy, curriculum, didactics, Educational Sciences.

\section{Introducción}

$\mathrm{E}$ 1 proceso formativo, tal como se ha planteado desde el siglo XVII y XVIII en las voces de Comenio (2012) y Herbart (1806), es adoctrinante. Es por ello que el antídoto contra la formación integral es la decolonialidad holística de la mente humana. Debemos transitar hacia la decolonialidad de la educación, lo cual implica aprender a caminar por los bordes, no temer movernos en la frontera. Si nos sumergimos en las teorías, enfoques y propuestas eurocéntricas de la educación, podríamos terminar ahogados en un mar de colonialidad epistémica-disciplinar. En cambio, si danzamos en la frontera con la opción decolonial, nuestro baile estará configurado por las acciones decolonizantes, conformadas por suficiente oxígeno para garantizar una respiración sana y una vida plena, libre de impurezas coloniales que laceran nuestro sistema nervioso central, nuestro cerebro, todo nuestro cuerpo, pero, sobre todo, nuestra mente, nuestra conciencia, nuestro pensar, nuestro hacer, nuestro sentir y nuestro vivir.

Para redireccionar y reposicionar las prácticas que emerjan de un carácter emancipatorio, es urgente ir descentralizando las teorías tradicionales e ir visibilizando otras perspectivas del conocimiento en lo que se enseña, las metodologías y la puesta en marcha de la didáctica. Es evidente que emergen una pedagogía, un currículo y una didáctica decoloniales. Así, el acto educativo cobrará importancia para la configuración del conocimiento, por medio de la inclusión de todos los actores sociales. Por ello, podemos afirmar que se avecina la pedagogía decolonial configurativa, promotora de un pensamiento decolonial/fronterizo, el cual gesta un pensamiento decolonial configurativo. Precisamente, este artículo está encaminado a pensar una pedagogía decolonial, que se inscribe en el movimiento de las pedagogías "otras", sustentada en las biopraxis pedagógicas decoloniales 


\section{Pedagogías "otras"}

Hoy en día no es posible realizar reflexiones sobre la pedagogía sin hacer referencia a las prácticas educativas o formativas, es decir, a las relaciones interpersonales que se establecen dentro y fuera del espacio áulico entre profesores y estudiantes, en las cuales los contenidos curriculares tienen una importancia extraordinaria. Es inútil que un profesor despliegue un excelente discurso sobre la inclusión, si se relaciona con sus estudiantes a partir de su etnia, estrato económico, orientación sexual, filiación religiosa o ideología política. En ocasiones, vemos profesores formadores de maestros desarrollando un discurso sobre el constructivismo pedagógico y sobre el enfoque histórico-cultural, haciendo elocuencia de la teoría piagetiana y los invaluables aportes de Vigotsky; sin embargo, estos docentes no permiten que sus estudiantes pregunten y solo ellos hablan, desarrollando una clase magistral, expositiva, sustentada en modelos pedagógicos tradicionales y enfoques conductistas. Es posible, también, ver docentes desarrollando clases sobre democracia e imponiendo su criterio sobre el pensar/sentir de sus estudiantes. Es preciso que los profesores reconozcamos la pluralidad y diversidad de formas de vivir, estar, ser, pensar de nuestros estudiantes, para no incurrir en estas acciones excluyentes en las que subyace la colonialidad en alguna de sus dimensiones:

La educación es la estrategia de colonialidad por antonomasia, de modo tal que es con ella que se siguen consolidando $-\mathrm{y}$ a veces con las mejores intenciones como son las campañas intensivas de alfabetización - el funcionamiento de la matriz colonial en la formación de los imaginarios, entendiendo por éstos las formas de percibir y comprender el mundo propias de cada cultura, siempre conflictivas y contradictorias. (Palermo, 2014, p. 45)

La pedagogía por excelencia, para atenuar lo anterior, es el ejemplo cotidiano de los profesores, su comportamiento ético, humilde, solidario y respetuoso. Un profesor que con su discurso pedagógico exprese disgusto por la diversidad de sus estudiantes o los trate con desprecio no merece ejercer esta noble y digna profesión. En ocasiones, escuchamos expresiones que maltratan y reprimen la pluralidad humana en las aulas. Es inverosímil que en pleno siglo XXI aún existan 
profesores quienes constriñen, desvalorizan e intimidan al estudiante activista de un movimiento juvenil, al no-blanco, al que profesa una religión, al que practica formas no normativas de sexualidad, al indígena, a la mujer o al afrodescendiente.

En todos esos casos señalados, el valor de la pluralidad humana no está siendo reconocido por el docente y, por lo tanto, no podríamos hablar aquí de una educación intercultural. Tampoco hay educación intercultural, si el Estado o el gobierno de turno imponen los contenidos curriculares sin permitir que los maestros realicen adaptaciones y contextualizaciones de estos, teniendo en cuenta las particularidades y singularidades de sus estudiantes:

La verdadera educación intercultural es aquella en la que el Estado se coloca como un interlocutor más, a través de la escuela y la universidad, y admite revisar, a partir del impacto de esta relación de intercambio que así se establece, su canon eurocéntrico: no hay interculturalidad sin descolonización activa de las prácticas educativas. (Palermo, 2015, p. 139)

Decolonizar la educación significa, entre otros argumentos, reconocer que los indígenas, campesinos, afrodescendientes o no-oyentes vienen a la universidad no solo a aprender y transformarse, sino también a enseñar. La decolonialidad de la educación se logra en la misma medida en que se reconoce la validez e importancia de los saberes "otros" no oficializados por la matriz colonial:

Esto implica modificar temas, contenidos, abordajes y estilos de transmisión, es decir, una serie de dimensiones de la educación que naturalizamos y, por lo tanto, consideramos inescapables; dimensiones que, sin embargo, resultan insoportables para una cantidad de gente que llega a la institución desde otros mundos, desde otras historias, con subjetividades divergentes forjadas a lo largo de otras trayectorias nunca debidamente acogidas ni representadas por el discurso estatal. (Palermo, 2015, p. 141)

La regularidad que emerge de estas reflexiones sobre la necesidad de una educación intercultural que respete la pluralidad y diversidad humana es la mordaz crítica al eurocentrismo de la educación 
latinoamericana en todos sus niveles, desde la primera infancia hasta la universidad. La solución no está relacionada con el diseño de nuevos currículos problematizadores ni con la aplicación de estrategias didácticas lúdicas como a veces se promociona. El tema es mucho más complejo. Urge "desmontar el horizonte eurocéntrico que circunda e impregna todos los aspectos y todos los niveles del quehacer educativo en nuestros países" (Palermo, 2015, p. 143).

La pedagogía moderna/occidental no puede reconocer ni visibilizar las diferencias por cuanto su interés estuvo centrado en homogeneizar, de ahí que sea una pedagogía colonizante. Frente a la diversidad/diferencia/lo otro/lo distinto/lo heterogéneo, la respuesta fue hacer que todo se parezca al modelo europeo colonial (Palermo, 2014). Esta es una pedagogía obsoleta, cuyos postulados epistémicos están en crisis, han caducado, y es por ello que los educadores debemos asumir los preceptos del giro decolonial, sumarnos a la resistencia y luchar por la configuración de una pedagogía "otra". Ahora bien, para pensar una pedagogía "otra" — no otra pedagogía - es necesario retar, desafiar, afrontar, hacerles frente abiertamente y resistir las diversas colonialidades que limitan nuestra existencia cotidiana: la colonialidad del saber, del poder, del ser y del vivir.

Walsh (2012a, 2012b, 2012c) considera que cuando enfrentamos la colonialidad del saber estamos creando las condiciones para superar el monismo en la definición de "la ciencia". De esta manera, crea los cimientos para un sistema educativo "otro", desde la educación en la primera infancia hasta la educación superior, desde la escuela hasta la universidad. Esta nueva concepción educativa puede $-\mathrm{y}$ debedesafiar la actual geopolítica dominante del conocimiento, retar sus orientaciones y racionalidades occidentales y USA-euro-céntricas, intentando pluralizar las epistemes configuradas, instauradas e impuestas desde el sistema-mundo moderno/colonial:

En América Latina esta geopolítica se evidencia sobre todo en el mantenimiento del eurocentrismo como perspectiva única, o al menos dominante, del conocimiento. Es una perspectiva presente en universidades, colegios y escuelas, que exalta la producción intelectual euroamericana como ciencia y conocimiento universales, relegando en forma oposicional el pensamiento del Sur - sea latinoamericano, africano o de los otros "sures" del 
planeta - al estatus de "saber localizado", negando el hecho de que el conocimiento producido en Europa y Estados Unidos también es local; su universalización al resto del mundo como algo obligatorio para todos es, en efecto, el problema central de la geopolítica del conocimiento y su legado colonial e imperial. (Walsh, 2009, p. 187)

En el campo de la educación y en la teoría pedagógica, también ha operado de manera silenciosa y exitosa esta colonialidad epistémica y epistemológica, negando otras formas ancestrales, diversas, singulares o emergentes de configuración de conocimiento, simplemente por no ser europeas, norteamericanas, blancas, patriarcales o "científicas" (desde la epistemología cartesiana-newtoniana). Esta racionalidad empirista-positivista separa el conocimiento del mundo, asumiendo una epistemología dualista fragmentadora, en la que el objeto de estudio existe "afuera" del sujeto y, por lo tanto, el conocimiento es interno y el mundo es externo. En contraposición a esta idea, Walsh (2012a) asume y nos exhorta a vivir una vida holística, integral, en la que el conocer y el vivir están estrechamente relacionados, configurados. Esta lógica que llamaremos holística-configuracional ubica el conocer y el proceso de este en un camino diferente al pensar instrumental, individual, que transita por una racionalidad medio-fin. La lógica holística del conocer transita en/desde/ por/para un pensamiento configuracional (Ortiz y Salcedo, 2014).

Los supuestos y postulados configurados desde esta lógica configurativa no son cerrados, rígidos y dogmáticos, sino abiertos y flexibles, involucran todos los sentidos y aptitudes del ser humano en el proceso de conocer, y no ponen límites al pensar ni al conocer. De esta manera, podríamos afirmar que el "buen vivir" no es solo praxiológico, social y económico, sino también epistémico (Walsh, 2012a).

Desde la epistemología configuracional (Ortiz, 2013, 2015, 2016a, 2016b, 2016c, 2016d, 2016e, 2016f, 2016g, 2016h) la visión sobre el universo, el cosmos, el mundo en que vivimos, nuestra propia práctica sociocultural y nuestras experiencias, vivencias, comprensiones y significaciones (biopraxis), no es una visión fragmentadora sino holística, integradora y relacional, en la que se integran, en un entretejido armónico y coherente, diversas configuraciones comprensivas: biológico-social, emoción-razón, espacio físico-conciencia, genético-cultural, interno-externo, material-espiritual, mente-cuerpo, objetivo-subjetivo, 
ser humano-naturaleza, sujeto-objeto, tangible-intangible, entre otras relacionalidades. Este entramado complejo puede ser analizado desde cuatro principios fundamentales: relacionalidad, correspondencia, complementariedad y reciprocidad (Walsh, 2009).

El vivir humano o Kawsay configura estos cuatro principios. Es decir, en "la experiencia plena de vivir, al gozo del sentido profundo de la vida, producto de la tensión armónica de las polaridades que emergen desde la convivencia intercultural armónica y respetuosa"5 (citado por Walsh, 2012a). De esta manera, "se enraiza en las relaciones equilibradas, armónicas, equitativas y solidarias entre humanos y con la naturaleza, en la dignidad de cada ser humano y en la necesaria interrelación entre seres, saberes, culturas, racionalidades y lógicas de pensar, actuar y vivir" (Walsh, 2012a, p. 71).

Lo anterior nos lleva a problematizar la episteme de la razón universal, debemos cuestionar la idea de una verdad global, estableciendo las bases para la configuración de otras racionalidades, verdades "otras" que desafien y enfrenten el caos ecológico (ambiental, espiritual, humano y social) que vivimos cotidianamente. Esto permite configurar nuevas formas de convivir en ciudadanía, diversidad y armonía, que visibilicen las cosmovisiones, epistemologías, filosofías, prácticas y maneras de vivir ancestrales, las cuales se sustentan en verdades y razones diferentes, en pensares, sentires, saberes, haceres y vivires "otros":

El buen vivir así abre las posibilidades de concebir y agenciar la vida de una manera "otra", una manera distinta concebida desde la diferencia ancestral y sus principios, pero pensada para el conjunto de la sociedad. Plantea, de esta manera, la posibilidad de un nuevo contrato social y una nueva razón decolonial enraizados en la relación y convivencia ética entre humanos y su entorno, con el afán de retar la fragmentación, promover la articulación e interculturalización y tejer una nueva identificación social, política y cultural de país que acepte las particularidades histórico-ancestrales a la vez que tome distancia de los designios del capitalismo global y su razón única para retomar - y reconstruir - la centralidad de Kawsay, es decir de la vida y del vivir. (Walsh, 2012a, p. 72)

5 http://www.amawtaywasi.edu.ec/objetivos.html 
El buen vivir riñe y agrieta la epistemología moderna/colonial, desde la cual nos informan - e imponen-que el conocimiento nos permite llegar al mundo. El buen vivir nos exhorta a asumir otra mirada epistemológica, que tiene sentido para la gran mayoría de los seres humanos: el mundo nos permite acceder al conocimiento, por lo tanto, no pienso, luego existo; más bien pienso según donde vivo. El vivir determina el pensar y no al revés como nos lo hace ver la epistemología eurocéntrica. Esto apunta a una epistemología, pedagogía y razón decoloniales (Walsh, 2006a, 2006b; 2008a, 2008b).

A partir de lo anterior, podemos afirmar que lo que necesitamos en la educación latinoamericana no es solamente reestructurar (González, 1998; Castro-Gómez, 2000), indisciplinar (Walsh, Schiwy y Castro-Gómez, 2002), reinventar/renovar (Santos, 2006a, 2006b), impensar/abrir (Wallerstein, 2010a, 2010b, 2011) o reconfigurar (Ortiz, 2016c) las ciencias sociales; lo que necesitamos es decolonizarlas, es decir, configurar unas ciencias sociales "otras", decolonizar la eurociencia y la europedagogía que nos ha colonizado desde hace más de cuatrocientos años, a partir del cogito ergo sum cartesiano, la didáctica "magna" de Comenio (2012), la pedagogía "general" de Herbart (1806), así como la concepción instrumental de currículum propuesta por Bobbitt $(1924,1941)$ y refrendada en Tyler (1986). Esta loable y urgente tarea de decolonizar la educación, las ciencias sociales y educativas, la pedagogía, el currículo y la didáctica no es posible solo desde la teoría y la pedagogía críticas. La teoría crítica (Horkheimer, 1998), en su formulación clásica de la Escuela de Frankfurt, y la pedagogía crítica, en sus postulados epistémicos originales, así como en las repercusiones de lo que McLaren (1997a, 1997b, 1998) llama pedagogía crítica "revolucionaria", son propuestas marxistas y antropocéntricas, esfuerzos occidentales que parten de un proyecto, pensamiento y supuestos paradigmáticos modernos/coloniales, a través de los cuales hacen crítica eurocéntrica al eurocentrismo.

Por otro lado, Grande (2008), en sus escritos sobre la "pedagogía roja", afirma que la pedagogía crítica revolucionaria sigue enraizada en el paradigma occidental, y, por tanto, en tensión con el conocimiento y la praxis indígena (Walsh, 2014a, 2014b, 2014c, 2014d, 2014e, 2014f). De ahí que las configuraciones conceptuales que permiten comprender la democratización, la propiedad y la subjetividad están definidas por configuraciones eurocéntricas que comprenden al ser humano como un 
sujeto primario de "derechos" y estatus social (Grande, 2008). Asimismo, en los Andes, Walsh (2014c) comenzó:

A ver la diferencia radical de un proyecto pensado desde y por los pueblos indígenas en el cual la cultura, la cosmología, la espiritualidad, la sabiduría, el conocimiento, la tierra, y la naturaleza $\mathrm{y} /$ como vida se entretejen con la autodeterminación, descolonización, movilización y transformación. (p. 21)

De esta manera, la colonialidad y la diferencia colonial deben ser abordadas por pedagogías "otras".

Estas pedagogías "otras" no han sido abordadas de forma abierta, directa y profunda por la modernidad ni por la teoría crítica, e incluso no están presentes de modo explícito en las obras de Freire $(1987,2011,2012 a, 2012 b, 2013 a, 2013 b, 2014)$ ni en ninguna de las obras de nuestro inmenso Martí (1961, 1975, 2007). Precisamente, Walsh (2014c) ha venido desplegando un distanciamiento de la pedagogía crítica, del pensamiento crítico occidental y de Freire, buscando y haciendo nuevos senderos, diferentes sendas y atajos, preguntando y caminando. En este mismo orden de ideas, Mignolo (2007a, 2007b, 2007c, 2008a, 2008b), desde el pensamiento decolonial, nos invita a asumir una opción decolonial que nos acerque al desprendimiento y la apertura epistemológica. Desprendernos de las ciencias de la educación y abrir la pedagogía implica dejar de caminar por las epistemes enraizadas en la epistemología moderna/colonial y comenzar a configurar nuevos caminos epistémicos y epistemológicos en los bordes de dichas epistemes, trayectos fronterizos por donde transitar decolonialmente. De ahí que nosotros diríamos como Walsh (2014c): nuestros senderos nos han hecho recelosos de la teoría crítica de occidente y nos han inducido a sospechar de "su ceguera a la colonialidad como el lado oscuro de la modernidad" (p. 23). Tenemos la impresión de que quienes critican la pedagogía tradicional aún están conectados a ella. Ni nosotros mismos estamos exentos de asumir en ocasiones sus postulados eurocéntricos colonizantes. Esto confirma lo arraigados que están en nosotros los supuestos epistémicos que se promulgan y la urgencia de impensar la pedagogía y las ciencias de la educación.

Desde la mirada esbozada anteriormente, consideramos que la pedagogía es una "verbalidad" (Vázquez, 2012), no es un simple 
sustantivo, sino un verbo, no es un ser (ontología), sino un hacer (praxiología). La pedagogía no es una simple disciplina o saber pedagógico, como la consideraba Zuluaga $(1987,2001,2003,2005)$. Tampoco Walsh (2014c) está interesada en la pedagogía como una disciplina; al contrario, aprecia su sentido y significado "en el accionar, en las prácticas, las metodologías, las estrategias y las maneras de hacer que se entretejen con - y son construidas en- resistencia y oposición, así como en insurgencia, afirmación y re-existencia, al imaginar y construir un mundo diferente" (p. 22). Esta autora postula una concepción diferente de la pedagogía, se compromete cada vez más a una búsqueda mayormente profunda, que incita a pedagogizar lo decolonial, y asume lo pedagógico desde una mirada praxiológica, la pedagogía como praxis, siguiendo los caminos de Freire (2011, 2012a, 2013b, 2014).

Según Walsh (2014a), "es oportuno pensar y significar hoy una perspectiva pedagógica —o de pedagogías - que se enlacen con los proyectos y perspectivas de interculturalidad crítica y decolonialidad" (p. 15). Pensamos que es el momento oportuno para la acción y el pensar decoloniales, si tenemos en cuenta los cambios que vive América Latina en los albores del siglo XXI:

Emergencia cada vez más enérgica y dinámica de intencionalidades, proyectos y procesos sustentados en $-\mathrm{y}$ encaminados $\mathrm{a}-\mathrm{la}$ decolonialidad.

Insurgencia, resistencia y (re)existencia epistémica, epistemológica, política y social.

Proliferación de movimientos y comunidades afro, indígenas, campesinos, mujeres y LGTBI.

Esta autora no incursiona en el proceso de decolonizar la pedagogía en tanto disciplina y ciencia de la educación, se concentra más en el proceso de pedagogización de las decolonialidades. Pensamos que, desde la mirada dialéctica configurativa, ambas vías son necesarias, urgentes e importantes: pedagogizar lo decolonial y decolonizar la pedagogía, sobre todo porque esta disciplina, al igual que lo ha demostrado Spivak $(2011,2015)$ sobre la filosofía, la literatura, la historia y la cultura occidentales, está atravesada por un pasado colonialista que aún no se ha superado y se reconfigura en nuestro presente postmoderno/ 
colonial. Bienvenida entonces la propuesta de Walsh (2014a), quien nos invita a configurar "pedagogía(s) de-colonial(es)".

\section{Hacia una pedagogía decolonial}

Según Palermo (2014), no hay "pedagogía" a secas, puesto que "la pedagogía sin modificador es una pedagogía oficial, al servicio del sistema político y económico que la sustenta, promueve y, también, la deja caer en pro de ocupaciones más "eficientes"' (p. 9). Asimismo, Esmeral y González (2015) integran la pedagogía y la interculturalidad, ofrecen criterios dialógicos de las realidades socioeducativas en el Caribe colombiano. Sin embargo, cuando hacen referencia al currículo del retorno, intentan integrar emancipación y decolonialidad, pero su análisis es insuficiente, no profundizan en la configuración epistémica de la decolonialidad y su potencial epistemológico, liberador y emancipador. De ahí que ampliamos y profundizamos el discurso de la decolonialidad de la educación como operación y acción tanto epistémica como epistemológica, para desprendernos del nocivo eurocentrismo instaurado desde el sistema-mundo capitalista/moderno/colonial. Pensar la decolonialidad de la educación se convierte así en la emergencia/urgencia de una pedagogía decolonial.

Walsh (2014a) no adiciona la pedagogía decolonial a la pedagogía crítica, ni sugiere lo decolonial como manifestación contemporánea de lo crítico. De hecho, afirma que:

La pedagogía decolonial tiene su propia genealogía - sin tener que necesariamente denominarla así- enraizada en las luchas y praxis que las comunidades afro e indígenas han venido ejerciendo desde años atrás, las que recientemente están saliendo a la luz y siendo reconstruidas y revitalizadas como parte de una postura y proyecto políticos. (p. 16)

Estos pensares, sentires y actuares integran y configuran tanto la pedagogía como la interculturalidad, desde una perspectiva $-\mathrm{y}$ en clave- decolonial, sin necesidad de transitar previamente por los postulados de la teoría crítica. 


\section{¿Qué — y cómo- son las pedagogías decoloniales?}

Pedagogías que promueven prácticas insurgentes de resistir, (re) existir y (re)vivir (Walsh, 2017).

Pedagogías que dialogan con los antecedentes crítico-políticos a la vez que parten de las luchas y praxis de orientación decolonial.

Pedagogías que enfrentan el monólogo de la razón moderno/ occidental/colonial y el mito racista de la modernidad/colonialidad (Bautista, 2009).

Pedagogías que visibilizan todo lo que el multiculturalismo oculta y lo que se escurre, incluyendo la teleología identitaria-existencial de la diferencia colonial, la geopolítica del saber y la topología del ser (Maldonado-Torres, 2006a, 2006b).

Pedagogías que configuran el cuestionamiento y el análisis crítico, la acción social transformadora, la insurgencia e intervención en los campos del poder, saber, ser y vivir; y, por último, que animan y asumen una actitud insurgente, es decir decolonial.

Pedagogías que transgreden, desplazan e inciden en la negación ontológica, epistémica y cosmogónica-espiritual que ha sido - y esestrategia, fin y resultado del poder de la colonialidad.

Pedagogías que abren grietas, desprendimientos y nuevos enganchamientos.

Pedagogías que provocan aprendizajes, desaprendizajes y reaprendizajes.

Pedagogías que aclaran y enredan caminos, no plantan dogmas o doctrinas, sino que siembran semillas para que puedan germinar de ellas conocimientos "otros" igualmente válidos a los saberes establecidos.

Pedagogías que configuran "horizontes de teorizar, pensar, hacer, ser, estar, sentir, mirar y escuchar - de modo individual y colectivohacia lo decolonial" (Walsh, 2013, p. 67).

Las pedagogías pensadas así no son externas a las realidades, subjetividades e historias vividas de los pueblos y de la gente, como parte integral de sus combates y perseverancias o persistencias, de sus luchas de concientización, afirmación y desalienación, y de sus bregas — ante la negación de su humanidad - de ser y hacerse humano. (Walsh, 2013, p. 31) 
Es decir, "la pedagogía o las pedagogías decoloniales serían ellas construidas y por construir: en escuelas, colegios, universidades, en el seno de las organizaciones, en los barrios, comunidades, movimientos y en la calle, entre otros lugares" (Walsh, 2014a, p. 15).

Las pedagogías decoloniales no son utopías (en el sentido que le atribuimos generalmente a esta noción como algo irrealizable) sino utopísticas (en el sentido que lo propone Wallerstein, 2003). Este autor considera la utopística como la evaluación seria, realista y profunda de las mejores alternativas, propuestas creativas, originales, novedosas y plausibles. La propone como un ejercicio simultáneo en los ámbitos de la ciencia, la política y la moralidad. Precisamente en este ámbito se inscriben las pedagogías decoloniales.

Todos los seres humanos que actualmente estamos viviendo en este convulso mundo, en este impredecible siglo XXI, ahora mismo, en este preciso momento, estamos configurados por el discurso de la modernidad/colonialidad en todas sus dimensiones: saber, poder, ser, vivir. De ahí que sea necesario pensar desde un lugar "otro", es imperiosa la necesidad de reflexionar desde la exterioridad de la episteme eurocéntrica (Palermo, 2014), para poder generar una pedagogía decolonial que haga frente al pensamiento único y universal, pero ¿cómo?:

El gran problema radica en cómo podrán los oprimidos, como seres duales, inauténticos, que "alojan" al opresor en sí, participar de la elaboración de la pedagogía para su liberación. Sólo en la medida en que se descubran "alojando" al opresor podrán contribuir a la construcción de su pedagogía liberadora. (Freire, 2012a, p. 42)

Desde esta mirada, las pedagogías decoloniales son sueños que se configuran en las biopraxis cotidianas de los seres humanos colonizados. Nunca se logran totalmente, los seres humanos subalternizados siempre estaremos luchando por conseguirla y nunca debemos abandonar nuestra "insurgencia social, cultural, política, epistémico-intelectual y educativa" (Walsh, 2014a, p. 16).

Siguiendo esta línea de pensamiento, Da silva (1999) enumera diversos discursos o propuestas educativas que nosotros hemos reformulado desde una postura metacognitiva en forma de interrogantes. Desde esta perspectiva, se impone la configuración de múltiples discursos pedagógicos decolonizantes. 
¿Para qué deseamos educar?

¿Educar para compensar, asimilar, reproducir o adoctrinar?

¿Educar para diferenciar o biculturalizar?

¿Educar para tolerar o prevenir el racismo y la exclusión?

¿Educar para transformar (pedagogía crítica)?

¿Educar para interactuar?

¿Educar para empoderar?

¿Educar para decolonizar?

¿Educar para comunalizar?

¿Educar para alterizar?

¿Cómo?

Desde estas reflexiones, damos el salto hacia una pedagogía decolonial, tratando de tener una mirada crítica, pero desde una perspectiva decolonial, transitando hacia la decolonialidad global o la globalización de la decolonialidad. Cada día nos convencemos más de la necesidad de decolonizar nuestro saber pedagógico, configurar una pedagogía decolonial y transitar en nuestras investigaciones sobre la educación hacia la decolonialidad epistémica y la desobediencia epistemológica. Desde esta mirada, apostar a una pedagogía decolonial es propender a desmantelar la "pedagogía de la crueldad" orientada a formar "sujetos dóciles al mercado y al capital" (Palermo, 2014, p. 136). Es evidente que en el campo de la educación es muy pertinente hoy el discurso sobre la decolonialidad. Por ejemplo, en la Universidad del Magdalena, Santa Marta, Colombia, que es una institución acreditada, no debemos trabajar para la reacreditación. Eso sería autocolonizarnos. La acreditación no es un fin, es un medio para alcanzar otros fines más trascendentales. La acreditación no es un resultado, es un proceso. Y nunca un resultado forma parte del proceso que lo genera. Si miramos solo el resultado y nos concentramos en él, entonces no vemos el proceso, que es lo más importante. No debemos trabajar para la reacreditación, debemos hacerlo para la decolonialidad de la educación y, si lo logramos, con toda seguridad vamos a recibir acreditación de manera permanente. Se necesita configurar una cultura de la excelencia, pertinencia, sentido y decolonialidad del vivir.

Illich (1974) propone una sociedad desescolarizada, y un poco con ironía, pero con seriedad en el primer epígrafe titulado ¿Por qué debemos privar de apoyo oficial a las escuelas?, plantea lo siguiente: 
Muchos estudiantes, en especial los que son pobres, saben intuitivamente qué hacen por ellos las escuelas. Los adiestran a confundir proceso y sustancia. Una vez que estos dos términos se hacen indistintos, se adopta una nueva lógica: cuanto más tratamiento haya, tanto mejor serán los resultados. Al alumno se le "escolariza" de ese modo para confundir enseñanza con saber, promoción al curso siguiente con educación, diploma con competencia, y fluidez con capacidad para decir algo nuevo. A su imaginación se la "escolariza" para que acepte servicio en vez de valor. Se confunde el tratamiento médico tomándolo por cuidado de la salud, el trabajo social por mejoramiento de la vida comunitaria, la protección policial por tranquilidad, el equilibrio militar por seguridad nacional, la mezquina lucha cotidiana por trabajo productivo. La salud, el saber, la dignidad, la independencia y el quehacer creativo quedan definidos como poco más que el desempeño de las instituciones que afirman servir a estos fines, y su mejoramiento se hace dependiente de la asignación de mayores recursos a la administración de hospitales, escuelas y demás organismos correspondientes. (p. 4)

Por estos días en Colombia (año 2018), en casi todas las universidades, se está llevando a cabo un amplio, fuerte y profundo debate sobre los resultados de las pruebas saber-pro. Casi todas las universidades colombianas hoy se enfrascan en la titánica y casi imposible tarea de diseñar e implementar estrategias que les permitan obtener resultados de excelencia en dichas pruebas por parte de los estudiantes. Ortiz (2018) ha hecho algunas reflexiones en las diversas reuniones metodológicas realizadas para analizar los resultados de las pruebas Saber Pro 2016, en la Facultad de Ciencias de la Educación de la Universidad del Magdalena (Santa Marta, Colombia). A continuación, sistematizamos lo que este autor denominó Decálogo para lograr que nuestros estudiantes tengan excelentes resultados en las pruebas estandarizadas, por cuanto entendemos que esta propuesta se inscribe en la perspectiva decolonial de la educación, la pedagogía, el currículo y la didáctica, aunque reconocemos que no necesariamente tienen que ser diez logos (decálogo), podrían ser siete u once, lo más importante no es el número ni la cantidad de propuestas, sino las reflexiones que estas pueden desplegar. 
a) No nos comparamos con otras universidades ni entre programas académicos.

Son seres humanos diferentes, por lo tanto, procesos inconmensurables. Una universidad puede estar en primer lugar y no haber avanzado. Otra universidad puede estar en último lugar y haber avanzado.

b) Ignoramos las pruebas, no pensamos en ellas, no las tenemos en cuenta, que las pruebas no nos quiten el sueño.

Mientras más pensemos y analicemos los resultados de las pruebas, menos posibilidades tendremos de revertir esos resultados. Porque nos perdemos en los resultados. Nos perdemos en los números y no vemos el proceso.

c) No preparamos a los estudiantes para las pruebas sino para el vivir.

Las pruebas no son ni siquiera parte de ese vivir. Una universidad puede tener primer lugar en las pruebas y no estar formando buenas personas, amorosas, responsables, respetuosas y solidarias. Esto no lo mide la prueba.

d) No pensamos en los resultados, nos concentramos solo en el proceso de formación.

Un resultado nunca es parte del proceso que le dio origen. Si me oriento al resultado nunca veo el proceso. Nos concentrarnos en el proceso y el resultado es una consecuencia de este. El resultado se deriva del proceso, pero no forma parte de él.

e) Reconocemos que los resultados de cualquier proceso evaluativo no dependen de la persona evaluada sino del evaluador y del instrumento evaluativo.

Si cambia el instrumento, también cambian los resultados. ¿Qué sucedería si en la próxima prueba el MEN cambia el estilo de las preguntas de la prueba?

f) Desarrollamos de manera constante y permanente, en cada una de las clases, cuatro acciones formativas: leer, escribir, reflexionar y conversar.

Leer, escribir, reflexionar y conversar. No hay otra solución más óptima y pertinente. Todo lo demás que hagamos serán paliativos, "pañitos de agua tibia".

Podemos desempeñarnos con veinticinco estudiantes por programa o con doscientos. Esa no es la esencia. La esencia es que en clases no leemos, no escribimos, no reflexionamos y no conversamos. Y esta es la ontología y la condición humana: leer, escribir, reflexionar y conversar. 
No hay aprendizaje auténtico y profundo sin leer, escribir, reflexionar y conversar. ¿Cuántos libros leen nuestros estudiantes en cada asignatura por cada semestre académico?, ¿qué autores leen?, ¿cuántas páginas escriben sobre lo leído?, ¿reflexionan en clases sobre lo leído y sobre lo escrito?, ¿conversan sobre un problema, tema o cuestión determinada relacionada con la asignatura?

Estas cuatro preguntas también aplican para nosotros los profesores. ¿Cuántos libros nos leemos al año?, ¿qué autores leemos?, ¿cuántas páginas escribimos al año, y de ellas cuántas publicamos?, ¿cómo desplegamos nuestro reflexionar en clases?, ¿conversamos en clases con nuestros estudiantes?, ¿estimulamos y potenciamos el reflexionar y el conversar entre ellos?

La lectura y la escritura son procesos compartidos, que se requieren con un fin determinado. En tal sentido, nuestros estudiantes deben saber y evidenciar para qué leen y qué hacen con lo que leen, qué hacen con las lecturas que sugieren los cursos de formación docente, por qué leen eso y no otra cosa. Pero, sobre todo, la generalización: qué escriben a partir de lo que leen, qué reflexión emerge de dicha lectura y sobre qué conversan a partir de esta. Ahora bien, la pregunta por la cantidad de libros que leemos en un año no indica ni sugiere que el aprendizaje depende de la cantidad de libros leídos, pero la respuesta a esta pregunta nada trivial sí devela qué estamos haciendo, qué no y qué debemos hacer para aprender, principalmente, desde configuraciones y matices decoloniales.

g) No desarrollamos todos los profesores de una misma asignatura la misma visión sobre el contenido curricular.

Si homogeneizamos el contenido, entonces limitamos el aprendizaje. El estudiante debe saber que existen distintas perspectivas, percepciones y concepciones sobre una misma cuestión. Además, no hay formación holística y desarrollo humano integral sin conflictos. La diversidad cognitiva configura contradicciones en los estudiantes, las cuales lo hacen pensar y reflexionar. Esto garantiza el aprendizaje auténtico y profundo.

h) Desarrollamos las clases sin hablar.

Mientras más habla el profesor en clases, menos aprenden los estudiantes. El profesor en clases no ofrece respuestas sino preguntas. Las preguntas son más importantes que las respuestas. La clase es una configuración heurística y hermenéutica, se desarrolla mediante un sistema de preguntas problematizadoras. No se aprende escuchando sino 
preguntando, cuestionando todo lo dado, problematizando. El preguntar, el reflexionar sobre lo preguntado y el conversar sobre lo reflexionado constituyen la configuración tríadica que garantiza la formación holística y el desarrollo humano integral. No hay aprendizaje sin conflicto cognitivo. Sin dilemas y tensiones intelectuales no se aprende. La contradicción engendra y genera el desarrollo humano integral.

i) No enseñamos desde una mirada ontológica sino epistemológica.

Es decir, no nos referimos a contenidos curriculares como si estos existieran de manera independiente a quien habla (mirada ontológica). El locus de enunciación es muy importante en la definición de los contenidos. Todos los contenidos existentes en la historia de la humanidad han sido dichos por alguien (mirada epistemológica)

j) No pensamos en lo que queremos cambiar en nuestros estudiantes, sino en lo que debemos conservar.

Ninguna acción ajena al estudiante determina su desempeño. Las estrategias didácticas tienen límites y estos los pone el estudiante. Cuando conservamos la configuración humana esencial e invariable, todos los demás procesos cambian en torno a las configuraciones que conservamos.

Partiendo de estas reflexiones, podemos afirmar que las organizaciones educativas no deben educar para superar un examen académico, sino para la vida. Definitivamente, el paradigma del "buen vivir" (Sumak Kawsay) o "vivir bien" (Suma Qamaña) y la configuración pedagógica de la felicidad basada en la pedagogía del amor (Ortiz, 2014) garantiza una educación para la vida, totalmente a la concepción desarrollista que nos han impuesto desde la modernidad/colonialidad. Es por ello que Walsh (2009) elabora la noción de pedagogía decolonial desde los principios del buen vivir, es decir, no solo desde criterios teóricos críticos, sino desde acciones prácticas de insurgencia educativa, lo cual se traduce en un proceso de creación, configuración e invención de nuevas condiciones culturales y de pensamiento, nuevos postulados y prácticas políticas y sociales. "Las pedagogías, en este sentido, son las prácticas, estrategias y metodologías que se entretejen con y se construyen tanto en la resistencia y la oposición, como en la insurgencia, el cimarronaje, la afirmación, la re-existencia y la re-humanización" (p. 29).

Hablar de un "orden del conocimiento" permite abordar el problema educativo desde otra perspectiva. Permite ir más allá de las políticas educativas o la propuesta curricular y considerar 
cómo la institución de la educación ha contribuido $-\mathrm{y}$ sigue contribuyendo - a la colonización de las mentes, a las nociones de "singularidad", "objetividad" y "neutralidad" de la ciencia, el conocimiento y la epistemología, y de que unas personas son más "aptas" — para pensar que otras. (Walsh, 2009, p. 186)

Siguiendo estas ideas, desde el grupo CRECED: Comunalidad de Reflexión sobre Colonialidad y Experiencias Decoloniales, hemos estado desplegando varias propuestas desde una perspectiva decolonial:

- $\quad$ Autodecolonialidad: altersofía —no epistemología - para la configuración de un pensamiento alterativo.

- Emergencia de un currículum decolonial en/desde el Sur: caracterización de las prácticas curriculares que reproducen una educación colonizante en las organizaciones educativas.

- Feminismo alterativo: una forma "otra" de sentir, conocer, pensar, aprender, ser y vivir.

- Interculturalidad decolonial: urgencia/emergencia de saberes "otros" en los procesos sociales y educativos.

- La decolonialidad en el contexto afrocolombiano y en las obras de Gabriel García Márquez y José Martí Pérez.

- Pensar la decolonialidad de la educación, la formación y la enseñanza: bases epistémicas para una pedagogía, un currículo y una didáctica decoloniales.

- Relaciones e intersecciones entre decolonialidad, alteridad y comunalidad.

- Tres décadas decolonizando: estado del arte de la investigación decolonial en/desde el Sur (1988-2018).

- Vigencia/pertinencia del pensamiento alterativo en/desde el Sur.

- Vocación decolonial desde las biopraxis pedagógicas.

Desde estas propuestas decoloniales, hemos realizado varias reflexiones, a partir del despliegue del hacer decolonial, práctica liberadora, emancipatoria y decolonizante, sustentada en tres acciones/ principios/criterios: observar comunal, conversar alterativo y reflexionar configurativo. A continuación, algunas preguntas que nos hemos hecho para pensar una pedagogía decolonial: 
¿Cómo se enseña y cómo se aprende en las organizaciones educativas? ¿Qué orientaciones didácticas predominan en el espacio áulico de las organizaciones educativas?

¿Cómo inciden los condicionantes institucionales y las geopolíticas del conocimiento en la formación de los maestros?

¿Qué rasgos caracterizan las prácticas pedagógicas dominantes?

¿Cómo se configura el currículo en las organizaciones educativas? ¿Cómo los docentes seleccionan los contenidos que enseñan?

¿Qué contenidos se enseñan?, ¿quién los configuró?, ¿cuándo?, ¿para qué y para quién?

¿Desde qué locus de enunciación se han configurado los contenidos que se enseñan?

¿De qué manera se podría configurar una ciencia sociohumana "otra" y una pedagogía "otra", que no reproduzcan la subalternización de subjetividades y saberes, ni el occidentalismo, eurocentrismo, colonialidad y racionalización "universal" de las ciencias hegemónicas; sino que se orienten a una mayor proyección e intervención epistémica y social decoloniales?

Los hallazgos parciales que se derivan del desarrollo de estas propuestas se encaminan hacia una reconfiguración de las ciencias sociales desde la decolonialidad de la educación. Impensar las ciencias sociales, abrirlas, configurar una nueva ciencia social para el siglo XXI, esto es lo que nos propone Wallerstein (2007a, 2007b, 2010a, 2011), quien devela los límites de los paradigmas decimonónicos y desentraña el eurocentrismo, a partir de analizar el universalismo europeo, el poder del discurso a través del discurso del poder (Wallerstein, 2007a). Este autor nos presenta un diagnóstico amplio, fino y profundo del sistema mundo, ofreciendo una exacta radiografía del capitalismo moderno/colonial (Wallerstein, 2010b). Desde esta mirada, estamos tratando de configurar una pedagogía, un currículo y una didáctica, desde perspectivas decoloniales. A partir de aquí, proponemos la noción de interculturalidad decolonial. Urge configurar un pensamiento de la decolonialidad. Por eso proponemos transitar desde la interculturalidad crítica hacia la interculturalidad decolonial configurativa.

El conversar decolonial nos ha hecho ver que el amor y la felicidad son pedagogías decoloniales útiles para el desarrollo del pensamiento configuracional en la formación holística. El tránsito hacia 
una pedagogía decolonial implica la reconfiguración de la educación del Sur. Necesitamos una epistemología de frontera: el decolonialismo o la decolonialogía como un paradigma epistemológico "otro", es decir, no otro paradigma superior al dominante en la actualidad, sino uno que coexista con el paradigma vigente. Urge transitar hacia una epistemología decolonial y una de la investigación decolonizante (Smith, 2013).

Debemos analizar la epistemología y la investigación en el siglo XXI, sus retos y desafíos decoloniales, hacer una crítica de la razón colonial y girar hacia una racionalidad decolonial. Para ello, es preciso pensar la pedagogía, el currículo y la didáctica desde una perspectiva decolonial, lo cual implica movernos desde la educación intercultural hacia la decolonial, renovar la educación, reinventar la pedagogía, decolonizar la formación, reconfigurar el currículo desde una mirada decolonial, decolonizar la enseñanza, reinventar la didáctica. Debemos educar para decolonizar, configurar una pedagogía de la decolonialidad. Es necesario configurar el currículo y la didáctica en clave decolonial, transitar hacia una enseñanza decolonial.

El aprendizaje decolonial es un imperativo en/desde el Abya-Yala. Debemos reconfigurar el perfil del profesor decolonial, delinear las características de la clase transmoderna, caracterizar las competencias decoloniales, proponer un modelo pedagógico decolonizante, que resignifique el rol del estudiante decolonizado, el del profesor decolonizante y reconfigurar la decolonialidad de la epistemología, la metodología de la investigación, la educación, formación, enseñanza, aprendizaje, pedagogía, currículo, didáctica. En fin, es un imperativo decolonizar la mente humana, el pensamiento, el lenguaje, la vida.

Como se aprecia, son inmensos los desafíos que tenemos, el reto de decolonizar la pedagogía, el currículo y la didáctica, mediante la ciencia decolonial, implica decolonizar la epistemología y la metodología de la investigación. Debemos formular un método de investigación decolonialógico, que nos permita configurar el currículo decolonial, la didáctica decolonial, el aprendizaje decolonizante, la enseñanza decolonial, la evaluación decolonial. La formación en el siglo XXI debe ser decolonizante. La decoloniagonía ${ }^{6}$ hoy nos mantiene inertes, hemos quedado perplejos ante la impotencia de ver la colonialidad en todos

6 Introducimos la noción de decoloniagonía con un doble sentido: es la agonía por el no logro de la decolonialidad global y es la visión de la decolonialidad, cómo la vemos y la asumimos desde la colonialidad que vivimos diariamente. 
los eventos y situaciones humanas, diluida como la sal en el mar, y no poder hacer nada para detener su afluencia arrolladora. ¿Será que el pensamiento de frontera, en tanto pensamiento configurativo decolonial, puede contribuir a la autodecolonialidad? ¿Cuál es el locus de enunciación costeño/caribeño/colombiano, que nos permita el logro de la decolonialidad holística?, ¿es posible configurar una inteligencia decolonial?, ¿cómo estimular la configuración del pensamiento decolonial en los niños? Esto solo es posible en las biopraxis pedagógicas decoloniales.

\section{Las biopraxis pedagógicas decoloniales}

Como ya hemos afirmado, Walsh (2009) concibe la pedagogía no como una ciencia ni como una disciplina académica, sino como una política sociocultural. Configurar esta noción proyecta una visión pedagógica que transita mucho más allá de los procesos de enseñanza y de aprendizaje, rebasa la concepción eurocéntrica de que la pedagogía está relacionada con la transmisión del saber, asumiéndola como "práctica social y política de producción y transformación, como modo de lucha crítica, dialógica y colectiva" (p. 16).

Walsh (2008a, 2013, 2014a, 2014c, 2014d) afirma que esta perspectiva pedagógica aún está en proceso de configuración, no solo desde el punto de vista teórico, sino desde el práctico. Cuando analizamos en profundidad esta novedosa y original propuesta, develamos la base de su inspiración y encontramos los principales referentes para su formulación en las prácticas educacionales de Freire (2012b, 2013a) y en las configuraciones teóricas aportadas por Fanón $(1965,2013,2016)$, sobre todo aquellas relacionadas con la conciencia del oprimido y la necesidad de humanización de los pueblos subalternizados. Walsh (2013) propone la configuración entre lo pedagógico y lo decolonial, a partir de la lectura de Freire, comprendiendo y asumiendo que son pedagogías, las cuales delinean caminos no solo para leer el mundo de manera crítica, sino con el afán de intervenir en la reinvención de la sociedad. Asimismo, desde Fanón, asume dichas pedagogías como acciones concretas que "avivan el desorden absoluto de la descolonización aportando una nueva humanidad" (p. 31).

En ocasiones, los gobiernos nacionales, regionales y locales, e incluso los directivos de organizaciones educativas, despliegan procesos 
de refundación educativa en el nivel del país, región o institución, ejecutando acciones reformistas desde concepciones sumativas y acciones algebraicas, adicionando diversidad cultural a las estructuras establecidas, pero sin realizar cambios estructurales profundos encaminados a reconocer e implicar las diferencias como elementos constitutivos de una pedagogía decolonial. De esta manera, se asumen las diferencias como diversidad cultural y no se reconoce el carácter colonial de estas; es decir, no se consideran diferencias coloniales que han emergido y se han desarrollado a partir de configuraciones jerarquizadas y naturalizadas que desde la época de la Colonia han asumido un estatus de inmanencia en la matriz de poder, conformando lo que hoy conocemos como colonialidad del poder. Es por ello que Walsh (2013) asume las pedagogías decoloniales como prácticas insurgentes de resistir, (re)existir y (re)vivir, es decir, pedagogías no como epistemes, teorías o saberes, sino como metodologías emergentes en los contextos de marginalización, subalternización, lucha, reexistencia y resistencia, "pedagogías como prácticas insurgentes que agrietan la modernidad/ colonialidad y hacen posible, maneras muy otras de ser, estar, pensar, saber, sentir, existir y vivir-con" (p. 19). Pedagogías como biopraxis pedagógicas decoloniales.

Estas reflexiones constituyen un intento de mostrar la recepción positiva que ha tenido la narrativa de la decolonialidad en Colombia y Latinoamérica, no solo como teoría y discurso, sino además como epistemología, acción y práctica en la educación. A estos eventos, acciones, $\mathrm{y}$ acontecimientos en los que subyacen intencionalidades formativas los hemos denominado "biopraxis pedagógicas decoloniales", las cuales surgen porque también existen prácticas colonizantes. Sin embargo, "toda praxis colonial, todo intento de dominación y de explotación inaugura irremediablemente un nuevo ciclo de conflictos en donde pueden observarse el despliegue simultáneo de praxis decoloniales" (Borsani y Quintero, 2014, p. 14).

Ortiz $(2017 \mathrm{a}, 2017 \mathrm{~b}, 2017 \mathrm{c})$ caracteriza la génesis del giro decolonial en las ciencias de la educación y valora la posibilidad de desarrollar biopraxis pedagógicas decoloniales. En este artículo llamamos biopraxis pedagógicas decoloniales a todas aquellas acciones críticas, desobedientes, emancipatorias, indisciplinadas, insubordinadas e insurgentes, liberadoras, obstinadas, resistentes y transformadoras, que emergen en el proceso formativo, dentro o fuera de los salones de clase, 
ya sea en espacios académicos como extraacadémicos, encaminadas a diluir la decolonialidad en dichos espacios, con el fin de configurar horizontes futuros de alternativas a la matriz de colonialidad.

Nos referimos a una praxis pedagógica crítica, intercultural y decolonial que pretende pensar no solo "desde" las luchas de los pueblos históricamente subalternizados, sino también "con" sujetos, conocimientos y modos distintos de estar, ser y vivir, dando un giro a la uninacionalidad y monoculturalidad fundadoras fundantes de la empresa educativa y su razón moderno-occidental-capitalista, para dar centralidad, más bien, a la vida y, por ende, al trabajo aún incompleto de la humanización y descolonización. (Walsh, 2012c, p. 176)

Ahora bien, nuestra acción decolonizante tiene dos dimensiones, una teórica-epistémica y otra praxiológica. Intentamos decolonizar la pedagogía en tanto saber, disciplina académica o ciencia, pero también intentamos decolonizarla en tanto praxis, en la cotidianidad del aula, en nuestro hacer. Como muy bien lo ha percibido Palermo (2014), a diferencia de los sociólogos, antropólogos, etnólogos, psicólogos y otros científicos sociales y profesionales de las humanidades, nuestro trabajo de campo se concreta en el aula de clases, en los procesos de enseñar, aprender y evaluar, "en tanto experiencia productora de saberes, participando con distintos sujetos portadores de memorias diversas, con experiencias vitales y dentro de sistemas de producción heterogéneos" (p. 63). Es decir, nuestras biopraxis pedagógicas decoloniales no se despliegan en/con/por/para poblaciones indígenas ni en/con/por/para comunidades de afrodescendientes, sino en el aula de clases, en/con/ por/para estudiantes de diversas etapas etarias, diversos ritmos y estilos de aprendizaje, diversos niveles instruccionales, heterogeneidad en su desarrollo en cuanto a sus competencias (conocimientos, habilidades, destrezas), diversa procedencia y, por supuesto, de diversas culturas, costumbres, aspiraciones, sueños, anhelos, miedos y esperanzas.

Las biopraxis pedagógicas decoloniales están conformadas por acciones formativas que se desarrollan en clave decolonial, es decir, desde una perspectiva o con un enfoque decolonial. Son todas aquellas acciones pedagógicas, curriculares, didácticas o evaluativas que tienen en cuenta "al otro", no lo subalternizan, no lo llevan a la periferia, ni lo 
abandonan en la frontera, sino que lo aceptan en la convivencia formativa, en un acto que solo es posible en el amar. Las biopraxis pedagógicas decoloniales se expresan, manifiestan y materializan en la configuración del currículo, o sea, en el diseño, desarrollo o evaluación curricular; y cobran vida en la didáctica: en el enseñar, en el aprender y en el evaluar.

El aula, a su vez, es un lugar insoslayable para propiciar la apertura a la contingencia y los procesos instituyentes de subjetivación que se articulan difusa y confusamente en él. En la medida en que logramos afirmar nuestra tarea docente en dicho terreno como una labor eminentemente problemática, nosotros mismos somos los puestos en cuestión. Y esto no sólo porque elegimos recorrer los temas desde el espacio y el tiempo que nos habilita el interrogante, sino ante todo porque nos arriesgamos cuando preferimos no legitimar nuestro discurso en la supuesta propiedad y transmisión de "verdades inclaudicables". Elegimos ponernos en cuestión a nosotros mismos, lo mismo que el mundo que representamos. Y esto es una parte sustantiva en la apuesta. (Aguer, 2014, p. 110)

Aguer nos está exhortando a problematizar, cuestionar y decolonizar nuestra propia práctica pedagógica, pero, ¿cómo se decoloniza una práctica pedagógica?, ¿cómo se despliegan las biopraxis pedagógicas decoloniales?

Cuando desarrollamos el hacer decolonial con docentes universitarios o de instituciones educativas, en la mayoría de los casos evidenciamos en las narraciones de sus experiencias, que ellos desarrollan acciones encaminadas a decolonizar sus prácticas pedagógicas, y lo hacen a partir de las operaciones siguientes:

Una explícita desobediencia a lo instituido, ya sea indisciplinando el conocimiento encerrado en asignaturas, ejecutando acciones que generan espacios de participación simétrica de los diferentes o incorporando tecnología comunicacional que aproxima a centros totalmente periféricos la actualidad del mundo en un proceso que se perfila como un proyecto glocal. Se trata de una articulación entre lo local y lo global que se retroalimentan en condiciones de defender lo local como fuerza política que utiliza la tecnología global en su propio beneficio. (Palermo, 2014, p. 108) 


\section{Conclusiones}

Podemos afirmar que la escritura de este artículo es en sí misma una práctica decolonizante, aunque no pretende ser una receta ni ofrecer orientaciones a los demás académicos. La decolonialidad de la educación es una opción que hemos asumido, pero somos conscientes de que no necesariamente deben asumirla otros docentes:

a. Configura un espacio/encuentro discursivo humanizante que cuestiona, critica y problematiza el discurso autoritario - sin ignorarlo- y lo enfrenta al conversar alterativo y el reflexionar configurativo como formas "otras" de aportar conocimiento colectivo.

b. Cuestiona y problematiza las jerarquías entre nosotros, dando lugar a una participación simétrica, activa y dialógica en la configuración de epistemes "otras"

c. Critica y cuestiona nuestras propias prácticas pedagógicas, convirtiéndose en una válida herramienta para la decolonialidad holística organizacional.

d. Incorpora la crítica y la autocrítica desde nuestro propio pensamiento decolonial y posicionamiento fronterizo, como resortes esenciales para dar sostenibilidad a proyectos de largo alcance que se reproduzcan en otros espacios académicos en los cuales participemos.

Finalmente, si nosotros queremos desplegar biopraxis pedagógicas decoloniales, debemos asumirlo como una opción personal, no transferible ni generalizable a todos los contextos. La decolonialidad de la educación no es una verdad absoluta, no es un conocimiento universal, es solo una opción, cuya intencionalidad es configurar un pensamiento decolonial.

Estas pedagogías decoloniales propician que los profesores desarrollemos nuestro pensamiento desde los bordes y la frontera, de ahí que las biopraxis pedagógicas decoloniales se despliegan a partir del desarrollo de un pensamiento fronterizo y un posicionamiento sustentado en la opción decolonial. Por lo tanto, las configuraciones pedagógicas, curriculares y didácticas no pueden ser universales, sino diversas, plurales y pluriversales. 


\section{Referencias}

Aguer, B. (Ed.). (2014). Cartografias del Poder y descolonialidad. Buenos Aires: Del Signo.

Bautista, R. (2009). Bolivia: Del Estado colonial al Estado Plurinacional. Bolivia: La Paz.

Bobbitt, F. (1924). How to make a curriculum. Cambridge: Riverside.

Bobbitt, F. (1941). The curriculum of modern education. New York: MacGraw-Hill.

Borsani, M. y Quintero, P. (Comp.) (2014). Los desafios decoloniales de nuestros días: pensar en colectivo. Neuquén, Argentina: Universidad Nacional del Comahue.

Castro-Gómez, S. (2000). La reestructuración de las ciencias sociales en América Latina. Bogotá: Centro Editorial Javeriano.

Comenio, J. A. (2012/1630). Didáctica Magna. Madrid: Akal.

Da silva, T. T. (1999). Documentos de identidad: Introducción a las teorías del currículum. Bello Horizonte: Auténtica Editorial.

Esmeral, S. J. y González, L. A. (2015). Pedagogía e Interculturalidad. Criterios dialógicos de las realidades socioeducativas en el Caribe colombiano. Bogotá: Kimpres.

Fanón, F. (1965). Racismo y cultura. En F. Fanon, Por la revolución africana (pp. 38-52). México: Fondo de Cultura Económica.

Fanón, F. (2013/1961). Los condenados de la tierra. Buenos Aires: FCE. Fanón, F. (2016/1952). Piel negra, máscaras blancas. Madrid: Akal.

Freire, P. (1987/1970). Justificativa da 'pedagogía do oprimido'. En P. Freire, Pedagogía do oprimido (pp. 16-32). Río de Janeiro: Paz e Terra.

Freire, P. (2011/1992). Pedagogía de la Esperanza. Un reencuentro con la Pedagogía del Oprimido. Madrid: Siglo XXI.

Freire, P. (2012a/1970). Pedagogía del Oprimido. Madrid: Siglo XXI.

Freire, P. (2012b/1993). Cartas a quien pretende enseñar. México: Siglo XXI.

Freire, P. (2013a/1969). La educación como práctica de la libertad. México: Siglo XXI.

Freire, P. (2013b/1984). La importancia de leer y el proceso de liberación. México: Siglo XXI.

Freire, P. (2014/1996). Pedagogía de la Autonomía. Saberes necesarios para la práctica educativa. México: Siglo XXI. 
González, P. (1998). Reestructuración de las ciencias sociales: Hacia un nuevo paradigma. Zona Abierta, 82(83), 267-285.

Grande, S. (2008). Red Pedagogy. The Un-Methodology. En N. Denzin, Y. Lincoln y L. T. Smith (Eds.), Handbook of Critical and Indigenous Methodologies (pp. 233-254). London: Sage.

Herbart, J. F. (1806). Pedagogía General derivada del fin de la educación. Madrid: Ediciones de la Lectura.

Horkheimer, M. (1998/1937). Teoría tradicional y teoría crítica. En M. Horkheimer, Teoría Crítica. Buenos Aires: Amorrortu.

Illich, I. (1974). La sociedad desescolarizada. Madrid: Barral.

Maldonado-Torres, N. (2006a). La topología del ser y la geopolítica del saber. Modernidad, imperio, colonialidad. En N. Maldonado-Torres, (Des)colonialidad del ser y del saber (pp. 63-130). Buenos Aires: Ediciones del Signo.

Maldonado-Torres, N. (2006b). Césaire's Gift and the Decolonial Turn. Radical Philosophy Review, 9(2), 111-137.

Martí, J. (1961). Ideario pedagógico. La Habana: Imprenta Nacional de Cuba.

Martí, J. (1975). Obras Completas. T. 18. La Habana: Editorial Ciencias Sociales.

Martí, J. (2007/1978). Obras Escogidas. Tres tomos. La Habana: Ciencias Sociales.

McLaren, P. (1997a). Pedagogía crítica y cultura depredadora. Políticas de oposición en la era postmoderna. Barcelona: Paidós.

McLaren, P. (1997b). Revolutionary Multiculturalism. Pedagogies of Dissent for the New Millennium. Boulder, Co.: Westview Press.

McLaren, P. (1998). Pedagogía, identidad y poder. Rosario: Homo Sapiens.

Mignolo, W. (2007a). Epílogo: Después de América y Postfacio a la edición en español: Después de América 'Latina', una vez más. En W. Mignolo, La idea de América Latina. La herida colonial y la opción decolonial (pp. 169-181, 201-219). Barcelona: Gedisa.

Mignolo, W. (2007b). El pensamiento decolonial. Desprendimiento y apertura. Un manifiesto. En S. Castro-Gómez y R. Grosfoguel (Eds.), El giro decolonial. Reflexiones para una diversidad epistémica más allá del capitalismo global (pp. 25-46). Bogotá: Siglo del Hombre.

Mignolo, W. (2007c). La idea de América Latina. La herida colonial y la opción decolonial. Barcelona: Gedisa. 
Mignolo, W. (2008a). La opción de-colonial: desprendimiento y apertura. Un manifiesto y un caso. Tabula Rasa, 8, 243-281.

Mignolo, W. (2008b). Género y descolonialidad. Colección Pensamiento Crítico y Opción Descolonial. Buenos Aires: Ediciones del signo.

Ortiz, A. (2013). Configuralogía. Paradigma epistemológico y metodológico en las Ciencias Humanas y Sociales. Barranquilla: Antillas.

Ortiz, A. (2014). Pedagogía del amor y la Felicidad. Bogotá: Ediciones de la U.

Ortiz, A. (2015). Epistemología y metodología de la investigación configuracional. Bogotá: Ediciones de la U.

Ortiz, A. (2016a). Epistemologías de la modernidad. Cambio de paradigma en el siglo XXI. Bogotá: Klasse.

Ortiz, A. (2016b). Humberto Maturana. Nuevos paradigmas en el siglo XXI. Psicología, Educación y Ciencia. Bogotá: Klasse.

Ortiz, A. (2016c). La Ciencia del tercer milenio. Hacia un nuevo paradigma epistemológico. Bogotá: EDIBERUM.

Ortiz, A. (2016d). La investigación según Humberto Maturana. El amor y la autopoiesis como epistemología y métodos de investigación. Bogotá: Magisterio.

Ortiz, A. (2016e). La investigación según Leonardo Da Vinci. Filosofía, Epistemología y Ciencia. Bogotá: Magisterio.

Ortiz, A. (2016f). La investigación según Niklas Luhmann. Epistemología de los sistemas y método sistémico de investigación. Bogotá: Magisterio.

Ortiz, A. (2016g). Niklas Luhmann. Nueva teoría general de sistemas. Bogotá: Klasse.

Ortiz, A. (2016h). Niklas Luhmann. Teoría emergente de los sistemas sociales. Bogotá: Klasse.

Ortiz, A. (2017a). Currículo y Didáctica. Curso desarrollado en el Doctorado en Ciencias de la Educación. Santa Marta, Colombia: Universidad del Magdalena.

Ortiz, A. (2017b). Decolonizar la Educación. Pedagogía, Currículo y Didáctica decoloniales. Barcelona: EAE.

Ortiz, A. (2017c). Decolonizar la investigación en educación. Praxis, 13(1), 93-104. Doi: http://dx.doi.org/10.21676/23897856.2112

Ortiz, A. (2018). Reflexiones realizadas en las diversas reuniones metodológicas para analizar los resultados de las pruebas Saber Pro 2016. Documento Inédito. Facultad de Ciencias de la Educación, Universidad del Magdalena, Santa Marta, Colombia. 
Ortiz, A. y Salcedo, M. (2014). Pensamiento Configuracional. Bogotá: REDIPED.

Palermo, Z. (Comp.). (2014). Para una Pedagogía decolonial. Buenos Aires: Del Signo.

Palermo, Z. (Ed.). (2015). Des/decolonizar la universidad. Buenos Aires: Del Signo.

Santos, B. de S. (2006a). Conocer desde el Sur. Para una cultura política emancipatoria. Lima, Perú: Editorial de la Facultad de Ciencias Sociales UMMSM.

Santos, B. de S. (2006b). Renovar la teoría crítica y reinventar la emancipación social. Buenos Aires: CLACSO.

Smith, L. T. (2013/1999). A decolonizar las metodologías. Investigación y pueblos indigenas. Chile: LOM.

Spivak, G. Ch. (2011/1988). ¿Puede hablar el subalterno? Buenos Aires: El Cuenco de Plata.

Spivak, G. Ch. (2015/1999). Crítica de la razón poscolonial. Hacia una historia del presente evanescente. Madrid: Akal.

Tyler, R. (1986/1946). Principios básicos del currículo. Buenos Aires: Troquel.

Vázquez, R. (2012). Towards a Decolonial Critique of Modernity. Buen Vivir, Relationality and the Task of Listening. En R. Fornet-Betancourt (Ed.), Capital, Poverty, Development, Denktradi-tionen im Dialog (pp. 241-252). Vol 33, Wissenschaftsverlag Mainz: Aachen.

Wallerstein, I. (2003/1998). Utopística o las opciones históricas del Siglo XXI. México D. F., México: Siglo XXI.

Wallerstein, I. (2007a). Universalismo europeo. El discurso del poder. México D. F: Siglo XXI.

Wallerstein, I. (2007b/1999). Conocer el mundo, saber el mundo: el fin de lo aprendido. Una ciencia social para el siglo XXI. México D. F.: Siglo XXI.

Wallerstein, I. (2010a/1991). Impensar las ciencias sociales. Límites de los paradigmas decimonónicos. México D. F.: Siglo XXI.

Wallerstein, I. (2010b/2004). Análisis de sistema mundo. Una introducción. México D.F.: Siglo XXI.

Wallerstein, I. (2011/1996). Abrir las ciencias sociales. México D. F.: Siglo XXI. 
Walsh, C. (2006a). Interculturalidad y (de)colonialidad: diferencia y nación de otro modo. En C. Walsh, Desarrollo e interculturalidad, imaginario y diferencia: la nación en el mundo Andino (pp. 2743). Quito: Academia de la Latinidad.

Walsh, C. (2006b). Interculturalidad y colonialidad del poder: Un pensamiento y posicionamiento otro desde la diferencia colonial. En C. Walsh, Á. García y W. Mignolo, Interculturalidad, descolonización del Estado y del conocimiento (pp. 21-70), Buenos Aires: El Signo.

Walsh, C. (2008a). Interculturalidad crítica / pedagogía decolonial. En A. Grueso y W. Villa (Eds.), Diversidad, interculturalidad y construcción de ciudad (pp. 44-63). Bogotá: Alcaldía Mayor de Bogotá y Universidad Pedagógica Nacional.

Walsh, C. (2008b). Interculturalidad, plurinacionalidad y decolonialidad: las insurgencias político-epistémicas de refundar el estado. Tábula Rasa, 9, 131-152.

Walsh, C. (2009). Interculturalidad, Estado, Sociedad. Luchas (de)coloniales de nuestra época. Quito: Abya-Yala.

Walsh, C. (2012a). Interculturalidad y (de)colonialidad. Perspectivas Críticas y políticas. Visao Global, Joacaba, 15 (1-2), 61-74.

Walsh, C. (2012b). La construcción del campo moderno del arte en el Ecuador, 1860-1925: Geopolíticas del Arte y Eurocentrismo. (Tesis Doctoral en Estudios Culturales Latinoamericanos). Universidad Andina Simón Bolívar, Quito, Ecuador.

Walsh, C. (2012c). Interculturalidad crítica y decolonialidad. Ensayos desde Abya Yala. Quito: Abya Yala.

Walsh, C. (2013). Pedagogías decoloniales: Prácticas insurgentes de resistir, (re)existir y (re)vivir. Tomo I. Quito: Abya-Yala.

Walsh, C. (2014a). Interculturalidad Crítica y Pedagogía Decolonial: Apuestas (des) de el In-surgir, Re-existir y Re-vivir. Recuperado de https://www.maxwell.vrac.puc-rio.br/

Walsh, C. (2014b). Notas Pedagógicas desde las Grietas Decoloniales. Quito, Ecuador: Universidad Andina Simón Bolívar.

Walsh, C. (2014c). Pedagogías Decoloniales. Caminando y Preguntando. Notas a Paulo Freire desde Abya Yala. Entramados-Educación y Sociedad, 1(1), 17-31.

Walsh, C. (2014d). Lo Pedagógico y lo Decolonial: Entretejiendo Caminos. Querétaro, México: Colectivo Zapateándole al mal Gobierno. 
Walsh, C. (2014e). Interculturalidad y colonialidad del poder. Un pensamiento y posicionamiento otro desde la diferencia colonial. En W. Mignolo (Ed.), Interculturalidad, descolonización del Estado $y$ del conocimiento. Buenos Aires: Del Signo.

Walsh, C. (2014f). Decolonialidad, interculturalidad, vida desde el Abya Yala-andino: notas pedagógicas y senti-pensantes. En M. E. Borsani y P. Quintero (Comp.), Los desafios decoloniales de nuestros días: pensar en colectivo (pp. 47-78). Neuquén, Argentina: Universidad Nacional del Comahue.

Walsh, C. (2017). Pedagogías Decoloniales. Prácticas insurgentes de resistir, (re)existir y (re)vivir. Tomo II. Quito: Abya-Yala.

Walsh, C., Schiwy, F. y Castro-Gómez, S. (Ed.). (2002). Indisciplinar las ciencias sociales. Geopoliticas del conocimiento y colonialidad del poder: perspectivas desde lo andino. Quito: Universidad Andina Simón Bolívar / Abya-Yala.

Zuluaga, O. L. (1987). Pedagogía e historia. La historicidad de la pedagogía. La enseñanza, un objeto de saber. Bogotá: Foro Nacional por Colombia.

Zuluaga, O. L. (2001). El saber pedagógico: experiencias y conceptualizaciones. En O. L. Zuluaga, Encuentros pedagógicos transculturales: desarrollo comparado de las conceptualizaciones y experiencias pedagógicas en Colombia y Alemania (pp. 81-88). Centro Consolidado de Investigaciones Educativas y Pedagógicas. Medellín: Editorial Marín Vieco.

Zuluaga, O. L. (2003). El pasado presente de la pedagogía y la didáctica. En O. L. Zuluaga. et. al., Pedagogía y Epistemología (pp. 61-72). Bogotá: Magisterio.

Zuluaga, O. L. (2005). Foucault: una lectura desde la práctica pedagógica. En O. L. Zuluaga, et. al., Foucault, la pedagogía y la educación. Pensar de otro modo (pp. 17-32). Bogotá: Magisterio. 
